\title{
AGO Recommendations for the Diagnosis and Treatment of Patients with Early Breast Cancer: Update 2018
}

\author{
Cornelia Liedtke $^{\mathrm{a}}$ Christian Jackisch $^{\mathrm{b}}$ Marc Thill ${ }^{\mathrm{c}}$ Christoph Thomssen ${ }^{\mathrm{d}}$ Volkmar Müller ${ }^{\mathrm{e}}$ \\ Wolfgang Jannif ${ }^{f}$ on behalf of the AGO Breast Committee*
}

${ }^{a}$ Klinik für Gynäkologie mit Brustzentrum, Charité - Universitätsmedizin Berlin/Campus Mitte, Berlin, Germany;

${ }^{b}$ Klinik für Gynäkologie und Geburtshilfe, Sana Klinikum Offenbach, Offenbach, Germany;

${ }^{c}$ Klinik für Gynäkologie und Geburtshilfe, Agaplesion Markus Krankenhaus, Frankfurt/M., Germany;

${ }^{d}$ Klinik für Frauenheilkunde und Geburtshilfe, Martin-Luther-Universität Halle-Wittenberg, Halle (Saale), Germany;

e Klinik und Poliklinik für Gynäkologie, Universitätsklinikum Hamburg-Eppendorf, Hamburg, Germany;

${ }^{f}$ Klinik für Frauenheilkunde und Geburtshilfe, Universitätsklinikum Ulm, Ulm, Germany

\section{Keywords}

Arbeitsgemeinschaft Gynäkologische Onkologie (AGO) . Early breast cancer . Systemic therapy · Local therapy . Treatment recommendations

\section{Introduction}

For almost 20 years, the Breast Committee of the Arbeitsgemeinschaft Gynäkologische Onkologie (German Gynecological Oncology Group/AGO) has been preparing and updating evidence-based recommendations for the diagnosis and treatment of patients with early and metastatic breast cancer. The AGO Breast Committee consists of gynecological oncologists specialized in breast cancer and interdisciplinary members specialized in pathology, radiological diagnostics, medical oncology, and radiation oncology. This update was performed according to a documented

${ }^{*}$ Members of the 'AGO Breast Committee' in alphabetical order: U.-S. Albert, Marburg; I. Bauerfeind, Landshut; J. Bischoff, Dessau; J.-U. Blohmer, Berlin; W. Budach, Düsseldorf; P. Dall, Lüneburg; I.J. Diel, Mannheim; N. Ditsch, Munich; P. Fasching, Erlangen; T. Fehm, Düsseldorf; M. Friedrich, Krefeld; B. Gerber, Rostock; V. Hanf, Fürth; N. Harbeck, Munich; J. Huober, Ulm; C. Jackisch, Offenbach; W. Janni, Ulm; H. Kreipe, Hanover; T. Kühn, Esslingen; S. Kümmel, Essen; C. Liedtke, Berlin; S. Loibl, Neu-Isenburg; H.-J. Lück, Hannover; D. Lüftner, Berlin; M. Lux, Erlangen; N. Maass, Kiel; V. Möbus, Frankfurt/M. V. Müller, Hamburg; M. Müller-Schimpfle, Frankfurt/M.; C. Mundhenke, Kiel; U. Nitz, Mönchengladbach; K. Rhiem, Cologne; A. Rody, Lübeck; M. Schmidt, Mainz; A. Schneeweiss, Heidelberg; F. Schütz, Heidelberg; P. Sinn, Heidelberg; C. Solbach, Frankfurt/M.; E. Solomayer, Homburg; E. Stickeler, Aachen; M. Thill, Frankfurt/M.; C. Thomssen, Halle (Saale); M. Untch, Berlin; F. Wenz, Mannheim; I. Witzel, Hamburg; A. Wöckel, Würzburg rule-fixed algorithm, by thoroughly reviewing and scoring, chapter by chapter, the recent publications for their scientific validity (Oxford level of evidence and grades of recommendation (LoE, GR)) [1] and clinical relevance (AGO recommendation (AGO); supplementary table 1). We present the 2018 update; the full version of the updated slide set is available online as a portable document format (PDF) file in both English and German [2].

\section{Options for Primary Prevention: Modifiable Lifestyle Factors}

Individual risk factors can be classified into non-modifiable, modifiable, and socially defined factors. Currently, there is good evidence that changes in some modifiable risk factors could substantially decrease the individual breast cancer risk.

Relevant lifestyle factors such as obesity, alcohol consumption, physical inactivity, smoking, and low fiber intake are well known. Furthermore, there is new data from a Dutch retrospective cohort study confirming that $25.7 \%$ of postmenopausal breast cancer cases are associated with lifestyle factors [3].

We would like to stress that obesity (high body mass index (BMI)) has a particularly significant influence on the incidence of primary and recurrent breast cancer. There is, however, uncertainty as to whether a high $\mathrm{BMI}$ is significantly associated with the diagnosis of triple-negative breast cancer (TNBC) [4]. Changing one's lifestyle has a preventive effect with regard to breast cancer: Maintenance of normal weight, fat-reduced diets, reductions in meat consumption and alcohol intake (particularly in case of estro-

\section{KARGER}

(C) 2018 S. Karger GmbH, Freiburg 
gen and progesterone receptor(ER/PR)-positive and/or invasive lobular tumors), smoking cessation, physical exercise, and avoidance of hormonal therapy (especially estrogen/progestin combination regimens) in postmenopausal women are controllable factors that may reduce the breast cancer risk.

\section{Breast Cancer Risk and Prevention}

Mutation analyses of BRCA1, BRCA2 and possibly other genes are currently being carried out on people with a family history of breast and ovarian cancer. A checklist facilitates the identification of persons with familial criteria for whom genetic counseling is an option. In addition, testing should be performed in the context of a therapeutic option (e.g., a poly(ADP-ribose) polymerase (PARP) inhibitor) as a consequence of data on the use of PARP inhibitors in $B R C A 1 / 2$ mutation carriers with advanced breast cancer [5]. Current data suggest that genes other than BRCA1 and BRCA2, which are real high-risk breast cancer susceptibility genes (LoE $2 \mathrm{a} / \mathrm{b} / \mathrm{A} / \mathrm{AGO}++$ ), are also associated with high risk of developing breast cancer [6]. However, before the widespread use of preventive measures, their effectiveness must be proven. Moreover, there are many non-BRCA-associated hereditary cancer syndromes with an increased risk for breast cancer (Li-Fraumeni syndrome, Cowden syndrome, hereditary diffuse gastric cancer syndrome, Peutz-Jeghers syndrome, Lynch syndrome). The penetrance of these genes depends on the family cancer history and the individual cancer load. The use of commercially available but not validated breast cancer gene panels for risk prediction is not recommended outside of prospective studies ( $L o E 3 a / B / A G O+/-$ ). Furthermore, clinical genetic testing for low-risk variants in clinical routine should be avoided (LoE 3b/D/AGO-). For many of these genetically defined subtypes, issues such as histopathological features, sensitivity to different screening modalities, course of disease, or specific treatment response still remain unclear.

In the context of gene panel testing, $20-30 \%$ of genetic test results reveal variants of unknown significance (VUS). Applying the classification of the International Agency for Research on Cancer (IARC), VUS are class 3 aberrations with a probability of being deleterious in $5-95 \%$ of cases [7]. Only class 4 and 5 variants (probability of $>95 \%$ and $>99 \%$, respectively) are clinically relevant. As more than $60 \%$ of the class 3 variants are extremely rare and population specific, only large databases such as that of the German Consortium of Hereditary Breast and Ovarian Cancer (GC-HBOC) or ENIGMA allow further classification of most of these variants and their publication in also publicly accessible databases such as clinvar.

Women with BRCA1/2 mutations should be offered nondirective counseling for the uptake of primary preventive measures (e.g., risk-reducing bilateral salpingo-oophorectomy after completion of family planning ( $\mathrm{LoE} 2 \mathrm{c} / \mathrm{B} / \mathrm{AGO}++$ ), risk-reducing bilateral mastectomy ( $\mathrm{LoE} 2 \mathrm{c} / \mathrm{B} / \mathrm{AGO}+$ ), or medical prevention with tamoxifen (LoE 1a/A/AGO+), raloxifen (LoE 1b/A/AGO+), or an aromatase inhibitor (AI) (LoE 1b/A/AGO+)) in addition to participation in an intensified surveillance program. Risk-reducing bilateral mastec- tomy after ovarian cancer is predominantly not indicated and could be thoroughly discussed depending on tumor stage (International Federation of Gynecology and Obstetrics (FIGO) I/II), recurrencefree time ( $\geq 5$ years) and age ( $<55$ years) (LoE $4 / \mathrm{C} / \mathrm{AGO}+/-$ ). However, uni- or bilateral mastectomy is not indicated in the absence of clearly defined genetic risk factors ( $\operatorname{LoE} 2 \mathrm{a} / \mathrm{B} / \mathrm{AGO}+$ ).

Data regarding the clinical benefit of risk-reducing contralateral mastectomy in affected BRCA1/2 mutation carriers suggested a disease-free survival (DFS) and overall survival (OS) benefit in specific subgroups only, particularly in patients aged $<40$ years with grade $1 / 2$ (G1/2) tumors, no TNBC, and no chemotherapy. Therefore, this intervention has to be thoroughly discussed with each individual patient (LoE $2 \mathrm{~b} / \mathrm{B} / \mathrm{AGO}+/-$ ). Breast-conserving therapy (BCT) is safe ( $\mathrm{LoE} 2 \mathrm{a} / \mathrm{B} / \mathrm{AGO}+$ ) and systemic therapy can be given according to recommendations for sporadic breast cancer (LoE $3 \mathrm{a} / \mathrm{B} / \mathrm{AGO}+$ ). The addition of carboplatin to neoadjuvant chemotherapy (NACT) seems to be beneficial for patients with TNBC regardless of their BRCA1 or BRCA2 mutation status [8]. Overall, the TNBC status in association with a BRCA1 or BRCA2 mutation displays an even higher overall chemotherapy sensitivity and better clinical outcome in comparison to patients without a BRCA1 or BRCA2 mutation.

To date, for the first time, there are treatment recommendations specific to $B R C A 1 / 2$ mutation carriers in the metastatic stage of their breast cancer. Based on data from the OlympiaD trial [9], PARP inhibitor monotherapy significantly prolongs the progression-free survival (PFS) in human epidermal growth factor receptor 2(HER2)-negative metastatic breast cancer patients with germline BRCA1/2 mutation compared to standard therapy (LoE 1b/A/AGO+). Moreover, in the advanced disease stage, carboplatin represents an effective treatment option favored over docetaxel in patients with breast cancer carrying a $B R C A$ mutation ( $\mathrm{LoE} 2 \mathrm{~b} / \mathrm{B} /$ $\mathrm{AGO}+$ ).

\section{Breast Cancer Diagnostics}

The aim of early detection and screening of breast cancer is to reduce breast cancer-specific mortality and treatment-dependent morbidity. Professionals and women need to be informed about the benefits and harms of cancer screening tests before making medical decisions. This includes clear and understandable information in absolute terms about false-positive rates (FPRs), falsenegative rates (FNRs), overdiagnosis, and overtreatment.

All available evidence confirms that mammography screening is capable of significantly reducing breast cancer mortality [10]. Based on a review by Oeffinger et al. [11], the number needed to screen (NNS) to prevent 1 breast cancer death with a mortality reduction of $20 \%$ (40\%) was estimated for women aged $40-49$ years to be 1,770 (753), for women aged 50-59 years 1,087 (462), and for women aged 60-69 years 835 (355). Screening mammography for breast cancer is recommended for women 50-74 years of age (LoE 1a/A/AGO++) [12-18]. For women 40-49 years of age, individual shared decision-making is recommended as these women would 
have smaller beneficial health effects than women of older age groups, e.g., higher recall rates for additional imaging and biopsy rates (LoE 1a/B/AGO+) [19-21]. There are no studies on women older than 75 years of age. However, in view of an aging population, screening can be offered to women in good health with a life expectancy of 10 years or longer ( $\mathrm{LoE} 4 / \mathrm{C} / \mathrm{AGO}+$ ).

For breast cancer screening, neither hand-held ultrasound (HHUS) nor automated whole-breast ultrasound (ABUS) alone are recommended ( $\mathrm{LoE} 3 \mathrm{a} / \mathrm{C} / \mathrm{AGO}-$ ). The arguments against ultrasound alone as a screening modality are lack of reproducibility, high FNR, low positive predictive value for biopsy, and lack of quality assurance.

The limitations of mammography are well known; hence, new technologies to overcome these constraints are highly welcome. Beside retrospective studies, 4 prospective studies within the European Breast Cancer Screening Program and 3 systematic reviews have been published. Digital breast tomosynthesis (DBT) added to 2-dimensional (2D) mammography proved to significantly increase the invasive cancer detection rate and reduced false-positive outcomes. In addition, first results on interval cancer rates showed a decrease. However, the radiation dose received with dual acquisition is increased [22-27].

DBT is particularly recommended if further mammographic diagnostic interventions like digital spot compression view are requested [28, 29] (LoE 2b/B/AGO+).

Mammography has a lower sensitivity and specificity in women with increased breast density, who also have a higher risk of breast cancer. To describe the mammographic breast composition, the detectability of masses affected by breast density (A-D) is recommended instead of the former categories using the percentage of glandular tissue (1-4) [30,31]. However, there is no gold standard for density determination as accuracy studies are missing. The recent (2016) systematic review by Melnikow et al. [32] revealed a reclassification rate of $12.6-18.7 \%$. The use of adjunct imaging techniques (DBT, HHUS, ABUS, magnetic resonance imaging $(\mathrm{MRI})$ ) in women with dense breast tissue increased the detection rate of cancers (rather than of ductal carcinoma in situ (DCIS)) for all imaging modalities. Harmful effects of supplemental imaging in women with dense breasts included higher recall and biopsy rates for women who do not have breast cancer. With DBT, the recall and biopsy rates were low ( $\mathrm{LoE} 2 \mathrm{~b} / \mathrm{B} / \mathrm{AGO}+$ ). Considering recent results of observational studies, HHUS struck the best balance on benefits and harms $[33,34](\mathrm{LoE} 3 \mathrm{~b} / \mathrm{B} / \mathrm{AGO}+)$.

To assess breast symptoms or lesions, clinical examination ( $\mathrm{LoE}$ 3b/B/AGO++), mammography (LoE $1 \mathrm{~b} / \mathrm{A} / \mathrm{AGO}++$ ), DBT where available (LoE 2b/B/AGO+), ultrasound (LoE 2b/B/AGO++), and minimal invasive biopsies ( $\mathrm{LoE} 1 \mathrm{c} / \mathrm{A} / \mathrm{AGO}++$ ) should be performed. Elastography (LoE 2b/B/AGO+) serves as an adjunct diagnostic modality and shows potential to decrease the false-positive biopsy rate.

Contrast-enhanced MRI plays an important role in acknowledged indications in diagnostic breast imaging as well as in highrisk patients. MRI should not be generally used to assess symptoms or breast lesions. MRI can be used if clinical examination, mam- mography, ultrasound and needle biopsy do not allow a definitive diagnosis (LoE 3b/B/AGO+). Second-look ultrasound is recommended in the case of lesions detected by MRI alone.

MRI should not be used in general for preoperative staging purposes in the case of BCT. According to a meta-analysis, the re-excision rate was not reduced. Furthermore, the initial and total rates of mastectomy increased if a preoperative breast MRI was performed compared with no preoperative breast MRI $[35,36]$. Preoperative breast MRI did neither help to reduce the rate of local recurrences nor to improve the local recurrence-free survival or distant metastasis-free survival [37]. It is important to realize that MRI-detected suspicious lesions should prompt MRI-based biopsy or marking for clarification. For some patients, e.g. those with reduced lesion detectability in mammography and ultrasound (detectability C-D), nipple involvement, lobular invasive cancer, suspicion of multilocular disease, and high risk, MRI can be considered ( $\mathrm{LoE} 1 \mathrm{~b} / \mathrm{B} / \mathrm{AGO}+/-)$ [38, 39]. The performance of MRIguided vacuum-assisted biopsies is mandatory if suspicious lesions are detected by MRI of the breast.

In the case of clinical and/or sonographic suspicious axillary lymph nodes, core needle biopsy is recommended to detect extensive axillary disease ( $\mathrm{LoE} 2 \mathrm{~b} / \mathrm{B} / \mathrm{AGO}++)$. Tagging the biopsied node with a titan clip may be helpful to identify it later; however, there is no agreement on the ideal technique ( $\mathrm{LoE} 3 \mathrm{~b} \mathrm{C/AGO+/-)}$ and study participation is recommended. The standard procedure in patients with clinically negative axillary lymph nodes is sentinel lymph node biopsy (SLNB).

Imaging for metastasis is recommended with computed tomography (CT) scan thorax/abdomen and bone scan in patients with high risk of metastasis (e.g. lymph node positivity) and/or symptoms, or if (neo-)adjuvant chemotherapy and/or HER2 treatment is indicated (LoE 2b/B/AGO+) [40].

\section{Pathology}

Little has changed with regard to routine pathological management of breast cancer.

When determining the ER status, it is recommended to recognize cancers with low receptor expression $(>1-10 \%)$ as a biologically distinct group. Breast cancers with borderline hormone receptor (HR) expression ( $>1$ to $<10 \%$ ) were initially regarded as HR-negative; however, today they are classified as HR-positive due to a change in the American Society of Clinical Oncology (ASCO)/ College of American Pathologists (CAP) guidelines. It has to be acknowledged though that the majority of data supporting this was published at a time when immunohistochemistry was not as standardized and sensitive as it is today. In contrast, recent publications [41-44] suggest that tumors with low ER expression share several features with TNBC, such as BRCAness, gene expression profiles, and prognosis. Therefore, it is recommended to define these cancers as 'low positive' rather than 'positive' in histology reporting. Nevertheless, the clinical consequences of this differentiation remain unclear. 


\section{Lesions of Uncertain Malignant Potential (B3)}

The group of lesions of uncertain malignant potential (B3) are typically detected in core or vacuum-assisted biopsy in asymptomatic women. The risk that is associated with B3 lesions cannot be strictly categorized according to the type of lesion (atypical ductal hyperplasia (ADH), flat epithelial atypia (FEA), lobular intraepithelial neoplasia (LIN), papilloma, radial scar), but additional clinical and pathological factors must be taken into consideration. The aim of further excision of B3 lesions is to detect more severe lesions, such as DCIS, which may be associated with a given $\mathrm{B} 3$ lesion, but also to minimize the risk of progression of a lesion of low malignant potential to an in situ carcinoma or invasive carcinoma [45].

$\mathrm{ADH}$ is characterized by a low-grade, monomorphic proliferation of atypical ductal epithelial cells which either partially or completely involve terminal ductal spaces but involve the ductal spaces to a total extent of less than $2 \mathrm{~mm}$ [46]. In a recent study on the long-term outcome of patients with $\mathrm{ADH}$ or lobular carcinoma in situ (LCIS) who underwent immediate re-excision after diagnosis on core needle biopsy, $30 \%$ of women had carcinoma or DCIS after the diagnosis of $\mathrm{ADH}$, and an additional $8 \%$ of these women subsequently developed carcinoma with a mean follow-up of 76 months. Women who had a benign immediate re-excision had a similar rate of $11.5 \%$ [47]. A somewhat lower rate of invasive carcinomas after follow-up of $\mathrm{ADH}$ after excisional biopsy was reported in historic data sets [48]. The reason for the high upgrade rate on open biopsy after the diagnosis of $\mathrm{ADH}$ is believed to be that $\mathrm{ADH}$ on core biopsy not infrequently represents inadequately sampled DCIS. In the same study, approximately three-quarters of the reported invasive carcinomas and DCIS after the diagnosis of ADH and a subsequent benign open biopsy occurred in the same breast.

The role of lobular neoplasia (LN) as a precursor lesion for invasive lobular carcinoma has been confirmed recently [49]. However, because of the low-risk nature of classical LN, a consensus has been reached that open excision can be avoided after the diagnosis of classical LN was established on core biopsy, and no discordant imaging, especially no focal lesion, is present $[50,51]$. This is supported by recent clinical data [52]. In contrast, high-risk variants of LN, which include pleomorphic and florid LCIS (pLCIS and fLCIS) are recommended for open biopsy, and preferably complete excision. Therefore, the pathologic distinction of classical-type LN from high-risk variants on core biopsy is most important [53].

A similar approach of conservative management avoiding open biopsy in the typical case of FEA has been recommended, provided that no mass lesion is present and imaging findings are concordant with pathologic findings [54]. An increased risk, and consequently a possible indication, has been suggested in cases of residual microcalcifications or with pure, prominent FEA following core biopsy $[55,56]$. Therefore, careful imaging-pathology correlation is recommended when considering treatment options in FEA [57].

The diagnosis of solitary or multiple papillomas on core biopsy carries a risk of approximately $10 \%$ for an invasive carcinoma or DCIS [58]. However, upgrade rates are widely different in the liter- ature and may be as low as 3.1\% [59]. It is important to distinguish papillomas that are associated with mass lesions from peripheral papillomas, which are often smaller but are commonly associated with proliferative breast disease. Clinical factors and imaging risk factors that are associated with increased risk include patient age, lesion multiplicity, and peripheral location. Significant risk factors on mammography include visibility and density, and on ultrasound, echo patterns, boundary and vascularity [60]. When papillomas were removed by vacuum-assisted core biopsies, no invasive cancer was seen on follow-up after 3.5 years. Therefore, conservative management is justified provided that the biopsy has been sufficiently representative and no discordance to imaging results was evident $[61,62]$.

A radial sclerosing lesion or radial scar may mimic carcinoma mammographically because of its stellate appearance. Radial sclerosing lesions are only rarely associated with atypia or DCIS [63]. Therefore, radial scars most often are benign lesions, and recent studies with careful radiological correlation have indicated that open biopsy may not be necessary for small lesions and for complete removal of the imaging abnormality [64-66].

In summary, there is accumulating evidence that open biopsy may be necessary only for a subset of patients with FEA, LIN, papilloma, or radial scar lesions, provided that careful radiologic-pathologic correlation was performed, no clinical, imaging, or pathologic high-risk factors are present, and that the imaging abnormality was completely or at least sufficiently representatively removed. This can often be achieved with a diagnostic-therapeutic vacuumassisted biopsy [67]. ADH is an exception and should be excised on most instances.

\section{Ductal Carcinoma in situ}

The diagnosis of DCIS increased dramatically following the introduction of screening mammography and comprises approximately $>20 \%$ of all newly diagnosed breast cancers [68]. However, epidemiological studies demonstrate that the removal of DCIS lesions has not been accompanied by a reduction in the incidence of invasive breast cancer $[69,70]$. The challenge of DCIS is to minimize the risk of overdiagnosis, to avoid under- or overtreatment and to prevent the development of invasive breast cancer. DCIS is commonly diagnosed by mammography, but up to $20 \%$ of DCIS remain mammographically occult due to the lack of calcifications and/or small tumor dimensions. The use of additional imaging techniques may theoretically be helpful to detect the full extent of a lesion and define surgical treatment. However, for the time being, the role of MRI in DCIS is limited. Breast MRI has a high sensitivity in the diagnosis of invasive breast cancer, varying from 90 to $100 \%$. The sensitivity for the diagnosis of DCIS is 77-96\% [71]. In summary, MRI does not improve the results of surgical therapy of DCIS [72, 73].

The biological characteristics of DCIS often predict recurrence and the type of invasive cancer that may develop in the future. Among patients with DCIS, the breast cancer-specific mortality 
was associated with age at diagnosis, ethnicity, grade, size, and ER status [74]. With appropriate risk prediction of subsequent development of invasive cancer, there is a better chance for individualized therapy. Breast cancer surgery (BCS) aims at the complete removal of the DCIS and represents the more favorable treatment in a majority of patients. Negative margins of at least $2 \mathrm{~mm}$ are associated with a reduced risk of ipsilateral breast tumor recurrence (IBTR) compared with positive margins defined as ink on DCIS. Negative margins of less than $2 \mathrm{~mm}$ alone are not an indication for mastectomy, and factors known to impact rates of IBTR should be considered in determining the need for re-excision $[75,76]$. The majority of trials reveal that, after surgery, adjuvant treatments reduce the rate of recurrent DCIS, and invasive recurrences have at the same time been unable to show an effect on mortality. Radiotherapy after BCS has been shown to halve both, in situ and invasive recurrences, in 5 phase III trials, 2 of which have also demonstrated that tamoxifen $20 \mathrm{mg} /$ day reduces the risk of ipsilateral and contralateral events by approximately $30 \%$ at both 10 and 15 years $(\mathrm{LoE} 1 \mathrm{a} / \mathrm{A} / \mathrm{AGO}+)[77,78]$. Omitting radiotherapy implies an elevated risk for local recurrence without an effect on OS even in the subset of 'good-risk' lesions. There remains a lack of level-1 evidence supporting the omission of adjuvant radiotherapy in selected low-risk cases such as those with tumors $<2.5 \mathrm{~cm}$, low and intermediate nuclear grade, and mammographically detected DCIS. Retrospective evaluation of the ER status showed that tamoxifen reduced any subsequent breast events by $42 \%$ in ER-positive DCIS $[79,80]$. AIs offer another endocrine option for postmenopausal women with ER-positive DCIS, and the choice between an AI and tamoxifen will probably depend more on the previous history of other conditions (e.g. osteoporosis and venous thrombosis) and short-term tolerability than on differences in efficacy. OS is not improved by endocrine therapy.

The optimal management in particular of adjuvant treatment and long-term risks must be discussed with the patients. Hence, the potential side effects of radiation therapy and endocrine therapy, albeit small, must be weighed more carefully when making treatment decisions for patients with DCIS.

\section{Prognostic and Predictive Factors}

Prognostic and predictive factors are an essential part of therapy concepts in early and advanced breast cancer. In 2018, the AGO guidelines for prognostic and predictive factors did not change substantially, since the data presented in 2017 altered the evidence levels (LoE) but not the AGO recommendations.

In HR-positive HER2-negative early breast cancer with 0-3 involved lymph nodes, gene expression assays may be used if established clinical pathological factors do not allow therapy decisions regarding the use of chemotherapy in addition to the standard of endocrine therapy. Patients with an estimated risk of recurrence of more than $10 \%$ at 10 years are generally considered candidates for upfront or adjuvant chemotherapy. The AGO recommends 4 tests $(\mathrm{AGO}+)$ that have been thoroughly validated retrospectively (LoE
IB for Endopredict ${ }^{\circledR}$, Prosigna ${ }^{\circledR}$ ) and prospectively (LoE IA for MammaPrint ${ }^{\circledR}$, Oncotype $\mathrm{DX}^{\circledR}$ ) for use in clinical routine. Prospective 5-year outcome data for Oncotype DX from the West German Study Group (WSG) PlanB trial [81] and for MammaPrint from the MINDACT trial [82] confirmed an excellent outcome in pN0-1 patients who had low-risk test results. Clinical outcome data from an analysis of a large prospectively designed registry investigating patients over a longer period confirm these results in real-life clinical practice [83]. Apart from the gene expression profiles, the tumor tissue concentrations of UPA/PAI1 (FEMTELLE ${ }^{\circledR}$ ) still have the highest levels of evidence (LoE 1aA/AGO+) with regard to identification of those patients with node-negative breast cancer who can avoid adjuvant chemotherapy for having a very low risk for recurrence. Moreover, pooled data suggests that high levels of these markers may predict benefit from chemotherapy $[199,200]$.

In HER2-positive early breast cancer, a meta-analysis $(n=967)$ demonstrates that pathological complete response (pCR) after neoadjuvant therapy (chemotherapy + anti-HER2 therapy) is significantly lower in phosphatidylinositol-4,5-bisphosphate 3-kinase catalytic subunit alpha (PIK3CA)-mutant versus wild-type tumors (16.2\% vs. $29.6 \%$; p < 0.001) (LoE IB). This difference was mostly due to a substantial difference in HR-positive tumors with pCR rates of $7.6 \%$ versus $24.2 \%$ ( $<<0.001)$; in HR-negative tumors, the numerical difference was not significant ( 27.2 vs. $36.4 \% ; \mathrm{p}=0.125$ ) [84]. Due to the lack of clinical consequences, there is no AGO recommendation for PIK3CA mutation analysis before NACT, so far $(\mathrm{AGO}+/-)$.

In triple-negative early breast cancer, the germline $B R C A$ status is predictive in response to NACT. In the neoadjuvant GeparQuinto trial, in 74 (15.8\%) out of 469 TNBC patients with available germline DNA, BRCA1 $(\mathrm{n}=61)$ or BRCA2 $(\mathrm{n}=13)$ mutations were detected. pCR (ypT0/ypN0) was observed in 50\% ( $\mathrm{n}=37)$ of the mutation carriers, but in only $31.1 \%(\mathrm{n}=123)$ of the patients without mutations $(\mathrm{p}=0.002)$. In patients without $B R C A$ mutations (hazard ratio $(\mathrm{HR})=0.20$; $95 \%$ confidence interval $(\mathrm{CI}) 0.11$ $0.34 ; \mathrm{p}<0.001)$ but not in mutation carriers $(\mathrm{HR}=0.48 ; 95 \% \mathrm{CI}$ $0.18-1.27 ; \mathrm{p}=0.129$ ), pCR (ypT0/ypN0) was significantly correlated with DFS [85].

As there are evidence-based consequences for patient management beyond the neoadjuvant therapy, germline BRCA determination is recommended in TNBC (LoE IIB AGO+). Yet, as shown earlier in the GeparSixto trial, the use of platinum should not depend on the germline BRCA status.

In patients with NACT, detection of $\geq 1$ circulating tumor cell (CTC) is an independent prognostic factor for locoregional relapse-free survival ( $\mathrm{HR}=1.8$; CI 1.2-2.7; $\mathrm{p}=0.001)$, distant DFS $(\mathrm{HR}=2.4 ; \mathrm{CI} 1.9-3.1 ; \mathrm{p}<0.0001)$, and $\mathrm{OS}(\mathrm{HR}=2.6$; CI 1.9-3.4; $\mathrm{p}$ $<0.0001)$. CTC positivity was not correlated with pCR in this meta-analysis comprising 2,156 patients from 21 studies [86]. Despite the high level of evidence ( $\mathrm{LoE} \mathrm{Ia} / \mathrm{B}$ ), the AGO does not recommend CTC detection in clinical routine due to the lack of therapeutic consequences (AGO+/-). 


\section{Breast Cancer Surgery under Oncological Aspects}

The extent of breast surgery has been consistently reduced in recent years. No ink on tumor is the accepted standard for resection margins for patients who undergo primary surgery or surgery after NACT (provided that all suspicious lesions according to preoperative imaging are resected $(\operatorname{LoE} 2 \mathrm{a} / \mathrm{A} / \mathrm{AGO}++$ and $\mathrm{LoE} 2 \mathrm{~b} / \mathrm{B} /$ AGO++, respectively)).

Although the role of axillary dissection (AD) is still under debate, especially in subsets of patients with clear indication for the adjuvant systemic treatment which is already defined by the intrinsic subtype of the tumor, the AGO clearly states that SLNB remains the standard of axillary staging for patients with invasive disease $(\mathrm{LoE} 1 \mathrm{~b} / \mathrm{A} / \mathrm{AGO}++)$. $\mathrm{AD}$ as a staging procedure has already been deleted in the 2017 guidelines (LoE 3/A/AGO-). In patients with 1-2 positive sentinel lymph nodes (SLNs) undergoing breast-conserving surgery as well as adequate systemic treatment and irradiation of the breast, completion of axillary lymph node dissection (ALND) is omitted in clinical practice. The American College of Surgeons Oncology Group (ACOSOG) Z0011 trial presented 10year follow-up data in 2016 with no differences in locoregional recurrences, DFS, and OS.

Due to limitations of the ACOSOG Z0011 trial and the lack of conformational studies, the AGO did not change the recommendation grade for $\mathrm{AD}$ in these patients $(\mathrm{LoE} 1 \mathrm{~b} / \mathrm{B} / \mathrm{AGO}+/-$ ) but recommends the participation in ongoing trials (e.g. INSEMA).

With regard to the technical aspects of the sentinel procedure, the role of presurgical lymphoscintigraphy (LSG) prior to SLNB was evaluated in a prospective multicentric randomized trial with 1,200 patients. The surgeons were randomized to know or not to know the results of the LSG before starting the SLNB. The number of removed sentinel nodes and the number of positive or negative lymph nodes were identical in both groups. Therefore, time, cost, and presurgical procedures can be optimized without LSG [92] (LOE $1 \mathrm{~b}$ (abstract) B/AGO+/-).

The role of SLNB in the neoadjuvant setting is still a matter of intense debate. Many clinicians agree that axillary staging after NACT would be more beneficial for the patient (reduction to one-step surgery, reduction of the $\mathrm{AD}$ rate due to conversion from $\mathrm{cN}+$ to $\mathrm{ycN} 0$, determination of $\mathrm{pCR}$ as an important prognostic parameter). However, data on the feasibility and reliability of SLNB in this setting were controversial, and data on regional recurrences are rare. The French GANEA II study, a prospective multicenter cohort study, enrolled 418 clinically node-negative patients who underwent SLNB alone after NACT. The detection rate for the SLN was $97 \%$. Only $1(0.2 \%)$ axillary recurrence was observed after 3 years. In a retrospective unicentric study, Galimberti et al. [201] published similar results. After 5 years of follow-up, only 1 (0.4\%) axillary recurrence was observed in 249 clinically node-negative patients who underwent SLNB after NACT. The AGO favors SLNB after NACT in cN0 patients (LoE 2b/B/AGO+). SLNB before NACT remains an option if an impact on adjuvant treatment decisions is expected (LoE $2 \mathrm{~b} / \mathrm{B} /$ $\mathrm{AGO}+/-)$.
For patients who present initially with (histologically proven) positive axillary lymph nodes ( $\mathrm{pN}+$ ), the feasibility and accuracy of SLNB is lower than in the adjuvant setting (SENTINA, ACOSOG 1071, GANEA I) [87-89]. Data on long-term outcome are insufficient, and it is unclear if the unfavorable FNRs translate into higher rates of recurrences. Therefore, the AGO still cannot generally recommend SLNB as standard procedure in clinically negative axillae after NACT (ycN0) in cases among whom tumor-infiltrated lymph nodes $(\mathrm{cN}+/ \mathrm{pN}+)$ have histologically been proven before NACT $(\mathrm{LoE} 2 \mathrm{bB} / \mathrm{AGO}+/-)$. Instead, $\mathrm{AD}$ may be a safer alternative in such cases (LoE $2 \mathrm{~b} / \mathrm{B} / \mathrm{AGO}+)$. Suggestions have been provided to improve the FNR for patients who convert from $\mathrm{cN}+$ to $\mathrm{ycN} 0$ after NACT. The 2018 AGO recommendations reviewed LoE for these candidates. An unplanned retrospective analysis of the ACOSOG Z1071 study revealed that the FNR could be improved when more than 2 SLNs were removed or when a dual tracer technique was applied. More than 2 SLNs were, however, identified in only $43.1 \%$ of the patients in the ACOSOG study and in $34 \%$ in the SENTINA trial. The recommendation of removing more than 2 SLNs would therefore be applicable in only an insufficiently small cohort of patients. It could furthermore motivate surgeons to remove additional non-SLNs and thus perform an undirected sampling (LoE $3 \mathrm{~b} / \mathrm{C} / \mathrm{AGO}+/-$ ). The improvement of the FNR by use of a dual tracer technique was not confirmed in the multivariate analysis of the SENTINA trial. Patients with a dual tracer had significantly more lymph nodes removed (3 vs. 2). The FNR was not significantly associated with the tracer technique ( $\mathrm{LoE} 3 \mathrm{~b} / \mathrm{C} / \mathrm{AGO}+/-)$. The prospective French FNAC study showed a significant correlation between the size of the lymph node metastases (defined as $\mathrm{pN}+$ ) and the FNR. When only patients with macrometastases were considered as $\mathrm{pN}+$, the FNR was $16.9 \%$. When micrometastases $(\mathrm{pN}+(\mathrm{mi}))$ or isolated tumor cells $(\mathrm{pN} 0(\mathrm{i}+))$ were considered as positive, the FNR dropped to 13.3 and $8.4 \%$, respectively [90] (LoE $2 \mathrm{~b} / \mathrm{B} / \mathrm{AGO}+/-)$. Therefore, the recommendations regarding pathological ultrastaging have been updated in this setting (LoE 2b/B/AGO+), especially with regard to ongoing treatments after NACT among patients without a pCR.

With the combined use of SLNB and a targeted removal of the biopsy-proven positive lymph node marked with a clip, seeds, or tattoo at the time of diagnosis ( $\mathrm{TAD}=$ targeted axillary dissection), the FNR can potentially be reduced [91]. Many issues including the surgical technique, the reproducibility of this retrospective unicenter study, and the extent of surgery are still unclear. Further studies (e.g. German SENTA, multicentric Tattoo) focusing on these issues are required before this procedure is introduced into routine clinical use (LoE 3b/C/AGO+/-).

\section{Oncoplastic and Reconstructive Surgery}

Oncoplastic surgery today represents an essential component in the framework of an integrated treatment strategy for patients with breast carcinoma. It is defined as the use of plastic surgery techniques at the time of tumor removal, in order to achieve safe 
resection margins, on the one hand, while on the other hand allowing an aesthetic breast shape [93]. Oncoplastic surcery focuses on favorable scar positioning, adequate soft-tissue shaping, the choice of a suitable reconstruction procedure (particularly when radiotherapy is indicated), and reconstruction of the contralateral side in order to achieve symmetry. The basic principles of reconstructive surgery $(\mathrm{AGO}++)$ require planning in an interdisciplinary tumor board prior to the actual surgical procedure itself. In general, the least burdensome surgical technique with an aesthetic result that will be stable over the longer term should be selected. The patient must receive detailed information and advice about all surgical techniques and their advantages and disadvantages, and about the option of obtaining a second opinion. In case of an unfavorable tumor/breast ratio, neoadjuvant systemic therapy might be considered, depending on the tumor biology. The preoperative counseling should include possible procedures for the contralateral breast if indicated. Contralateral procedures and subsequent operations in order to achieve symmetry should therefore be discussed with the patient even before the first operation. These operations are usually performed as secondary procedures after an interval of at least 3-6 months. The effects of radiotherapy on the affected side must be taken into account (e.g. volume reduction). Importantly, adjuvant therapy should not be delayed by breast reconstruction.

When mastectomy is necessary, skin-sparing techniques with complete resection of the breast tissue in suitably selected patients are associated with a similar recurrence rate and a better quality of life (LoE 2b/B/AGO++).

Depending on the location of the tumor, the mammillary and areola complex can also be preserved. The same also applies to prophylactic mastectomy (LoE 2b/C/AGO+).

Following a mastectomy, reconstruction can be carried out using pedicled or free tissue transfer, and also with the use of silicone gel-filled implants (LoE $2 \mathrm{a} / \mathrm{B} / \mathrm{AGO}+$ ). A recent analysis including 55,279 patients reported equal oncological safety compared to saline implants [94]. Reconstruction can be carried out either immediately or after an interval (LoE $3 \mathrm{~b} / \mathrm{B} / \mathrm{AGO}++)$. The latter does not delay any necessary adjuvant therapies, but may result in shrinkage of the skin cover. When free tissue transfer is being used, the deep inferior epigastric perforator (DIEP) or free transverse rectus abdominis muscle (TRAM) flaps are available (LoE 2a/B/ $\mathrm{AGO}+$ ). Both procedures are potentially muscle sparing, but the DIEP is associated with a lower rate of herniation.

In case of heterologous reconstruction, radiotherapy should be completed before using implants, to avoid a negative outcome (LoE 2a/B/AGO+) [95]. However, the patient must receive information about a high complication rate (e.g., capsule contraction, revision operations, failure of the reconstruction, impaired cosmetic appearance) and about the lower level of patient satisfaction in comparison with autologous reconstruction plus radiotherapy (LoE 2b/B) $[96,97]$.

In cases with indication for meshes, autologous tissue flaps (e.g. de-epithelialized corium-fat flaps), acellular dermis, or synthetic mesh are available and of equivalent value (AGO+). Volume defi- cits and scars can be corrected using lipotransfer both after breast preservation and after mastectomy with reconstruction (LoE 2a/B/ AGO+). Numerous studies confirming the oncological safety of these approaches have been published in the meantime [98].

\section{Neoadjuvant Chemotherapy}

Survival rates are similar after primary systemic ('preoperative', 'neoadjuvant') chemotherapy (NACT) and adjuvant therapy [99]. pCR defined as ypT0 ypN0 or ypT0/is ypN0 is associated with improved survival $[100,101]$. NACT remains the preferred therapeutic option in patients who have an indication for adjuvant chemotherapy (LoE 1/B/AGO++). A recent Early Breast Cancer Trialists' Collaborative Group (EBCTCG) meta-analysis of NACT trials with a follow-up of 15 years detected an increased rate of breast conservation with equal DFS and OS (compared to adjuvant therapy). The increased rate of local recurrence cannot be attributed to NACT, but to different rates of recurrence after BCS versus mastectomy [102]. Modern regimens are considered to be more efficient (higher pCR) and to be safe for patients (improved surgical and radiotherapeutical approaches). NACT allows responseguided therapies and novel post-neoadjuvant therapies in low or non-responding tumors. Dose-dense therapy regimens should be preferred $(\mathrm{AGO}++)$. In particular, in patient subgroups among whom a pCR is strongly associated with improved survival such as in TNBC, HER2-positive, and luminal B-like (HR-positive/HER2negative/G3, high Ki-67) cancer, NACT (plus targeted therapy) should be the preferred therapeutic approach $(\mathrm{AGO}++)$. In patients with TNBC (regardless of germline BRCA1/2 mutation status), a platinum-containing regimen may be considered ( $\mathrm{LoE} 2 \mathrm{~b} / \mathrm{B} /$ $\mathrm{AGO}+$ ) based on data from phase II randomized trials (e.g., GeparSixto, Cancer and Leukemia Group B (CALGB) 40603) [103]. The addition of carboplatin was not only associated with an increased pCR rate in both neoadjuvant trials but also resulted in a significant improvement in GeparSixto with a DFS rate of $85.8 \%$ (with carboplatin) versus $76.1 \%$ without carboplatin (HR 0.56; $\mathrm{p}=$ 0.0350 ) and a clinically meaningful albeit statistically not significant improvement in DFS (absolute 5\%) in the CALGB 40603 study. Furthermore, the results of the GeparSepto trial suggest particular benefit from using nanoparticle albumin-bound (nab)-paclitaxel $125 \mathrm{mg} / \mathrm{m}^{2}$ weekly instead of paclitaxel for patients with TNBC, which was not observed in the ETNA trial (LoE 2a/B/ AGO+/-) [104-107].

For HER2-positive patients, HER2-directed therapy is standard as part of neoadjuvant therapy. Given the significant increase in pCR rates and the trend for improved PFS observed in the neoadjuvant NeoSphere trial with the addition of pertuzumab to trastuzumab, dual blockade is highly recommended in patients at high risk for recurrence [108-111] ( $\mathrm{LoE} 2 \mathrm{~b} / \mathrm{B} / \mathrm{AGO}++)$.

In patients with $\mathrm{N}+$ or $\mathrm{HR}-/ \mathrm{HER} 2 / \mathrm{neu}+$ tumors, completion of combined therapy with trastuzumab and pertuzumab after surgery for a total of 52 weeks can be considered ( $\mathrm{LoE} 2 \mathrm{~b} / \mathrm{B} / \mathrm{AGO}+$ ). With respect to endocrine neoadjuvant therapy, in exceptional situa- 
tions, endocrine treatment with gonadotropin-releasing hormone $(\mathrm{GnRH})$ analogues plus an AI may be considered for premenopausal women (LoE $1 \mathrm{~b} / \mathrm{C} / \mathrm{AGO}+/-$ ). Response-guided treatment has been shown to be beneficial within the GeparTrio trial. Consequently, in the case of response after 2 cycles of DAC (docetaxel, adriamycin, cyclophosphamide) in HR-positive breast cancer, a total of 8 instead of 6 cycles of DAC may be considered to be appropriate (LoE $2 \mathrm{~b} / \mathrm{C} / \mathrm{AGO}+$ ). In the case of no response after $2 \mathrm{cy}$ cles of DAC, continuation of NACT with a non-cross-resistant regimen $(\mathrm{LoE} 2 \mathrm{~b} / \mathrm{B} / \mathrm{AGO}+)$ such as $4 \times$ vinorelbine/capecitabine (LoE 1b/B/AGO+) may be beneficial [112]. This can be an option in individual cases but cannot be considered as a routine approach. Post-neoadjuvant concepts are currently being investigated in clinical trials, and trial participation is recommended if possible, particularly in the case of no pCR. Given the positive results of the CREATE-X study, further chemotherapy in the case of no pCR can be considered in high-risk cases (capecitabine in TNBC) (LoE 2b/B/AGO+) [113]. Novel predictive factors, such as tumor-infiltrating lymphocytes (LoE I/B/AGO+) or PIK3CA mutation in the tumor (LoE II/B/AGO+/-), are promising tools but currently not recommended in the routine clinical setting [114-117]. Patients with germline $B R C A$ mutations have a higher probability to achieve a pCR [80]. The indications for mastectomy after NACT remain unchanged: positive margins after repeated excisions (LoE $3 \mathrm{~b} / \mathrm{C} / \mathrm{AGO}++$ ), lack of feasibility of radiotherapy (LoE 5/D/ $\mathrm{AGO}++$ ), and presence of inflammatory breast cancer (with no more than clinical complete response, LoE $2 \mathrm{~b} / \mathrm{C} / \mathrm{AGO}+$ ). In inflammatory breast cancer with pCR after NACT, BCT may be discussed with the patient. Furthermore, large tumors (cT4a-c) at first diagnosis represent only a relative indication for mastectomy after NACT (LoE 2b/B/AGO+/-). In the case of nodal involvement, biopsy and clip marking of the positive nodes is recommended if possible ( $\mathrm{LoE} 5 / \mathrm{D} / \mathrm{AGO}+$ ). The SLN procedure is recommended preferably after neoadjuvant therapy in the case of $\mathrm{cN} 0$ (LoE 2b/B/AGO+) (see also the section on surgery) [118-121]. Delayed initiation of NACT for thorough diagnosis (imaging and/or molecular pathology) is not correlated with a negative outcome (LoE 2b/B122). Surgery after NACT should be planned no later than 2-4 weeks after the last chemotherapy cycle, after patients have recovered from hematological toxicities $(2 \mathrm{~b} / \mathrm{B} / \mathrm{AGO}++)$ [122-124].

\section{Adjuvant Endocrine Therapy}

Endocrine therapy still remains one of the most effective treatment options in all settings of breast cancer. In the adjuvant situation, endocrine therapy is indicated in all patients with HR-positive breast cancer, as well as those with low HR-positive cancer $(\geq 1-$ $9 \%$, so-called 'questionably sensitive' cases; LoE 1/GR A/AGO++). If chemotherapy is indicated, endocrine therapy starts in sequence after cytotoxic therapy. At present, in the adjuvant setting, we distinguish between 'initial therapy' (years $0-5$ ) and 'extended adjuvant therapy' (EAT, years 6-15; AGO++). Treatment duration of 5 years remains the standard of care. EAT might be indicated in patients with increased risk of relapse based on the individual risk/ benefit ratio. Data from the EBCTCG estimates the risk of relapse after termination of endocrine adjuvant therapy at 5 years [125]. In premenopausal and perimenopausal patients, treatment with tamoxifen is indicated for 5-10 years (LoE 1a/GR A/AGO++). In accordance with data from the ATLAS and aTTom trials, tamoxifen therapy can be extended for up to 10 years [126]. If the patient is confirmed to be postmenopausal within the first 5 years, according to the data from the MA.17 study, endocrine therapy can be continued after 5 years of tamoxifen with 2.5-5 years of letrozole (LoE 1b/GR B/AGO+) [127].

If chemotherapy was indicated as part of the (neo-)adjuvant setting and ovarian function has recovered within the first 8 months afterwards, treatment with a GnRH analogue plus tamoxifen (LoE 1b/GR B/AGO+) or with the AI exemestane for 5 years can be considered on an individual basis ( $\mathrm{LoE} 1 \mathrm{~b} / \mathrm{GR} \mathrm{B} / \mathrm{AGO}+/-$ ). In patients under the age of 35 years, in accordance with the full publication of the SOFT study, a combination of tamoxifen with a GnRH analogue should be recommended, due to the significant benefit relative to $\mathrm{OS}$ ( $\mathrm{LoE} 1 \mathrm{~b} / \mathrm{GR} \mathrm{B} / \mathrm{AGO}++$ ) [128]. In women with endocrine-sensitive breast cancer without adjuvant chemotherapy and preserved ovarian function, the outcome of endocrine therapy with tamoxifen alone is highly favorable ( $\mathrm{LoE} 1 \mathrm{~b} / \mathrm{GR} \mathrm{B} / \mathrm{AGO}++$ ). The effect of additional ovarian downregulation with a $\mathrm{GnRH}$ agonist $(\mathrm{GnRHa})$ is limited for tamoxifen + GnRHa (LoE 1b/GR B/ $\mathrm{AGO}+/-)$ or AIs + GnRHa (LoE 1b/GR B/AGO+/-). However, increased side effects may impair compliance when GnRHa are added [129]. Tamoxifen alone still remains the standard of care.

It should be stated that GnRHa monotherapy might be indicated in case of contraindication for either tamoxifen or aromatase inhibition compared to no endocrine therapy at all (LoE 1a/GR B/ $\mathrm{AGO}+$ ).

In postmenopausal women, various options of the initial endocrine therapy (years 0-5) can be considered and there has been extensive discussion about the use of tamoxifen in comparison with an AI or sequential use of tamoxifen and an AI. Two metaanalyses have been published during the last year and both suggest that AIs should be preferred to tamoxifen [130, 131]. In the EBCTCG meta-analysis, 5 years of treatment with an AI led not only to an improved 10-year breast cancer mortality rate in comparison with 5 years of tamoxifen but also to a reduced rate of recurrences. Sequential treatment with tamoxifen followed by an AI was also superior with regard to mortality, so that in postmenopausal patients either an AI or sequential treatment with tamoxifen followed by AI, or vice versa, should be used (LoE 1a/GR A/ $\mathrm{AGO}++$ ). The 5-year AI therapy is preferable, particularly in patients with lobular cancer or with a high risk of recurrence, mostly if adjuvant chemotherapy was indicated. In summary, based on the EBCTCG publication in 2015, the most important impact on outcome was demonstrated when AIs were included for 3 years in the adjuvant setting. In postmenopausal women with low risk of recurrence, tamoxifen therapy upfront is still an option (LoE 1a/ GR A/AGO+). 
The issue of whether EAT should be recommended for recurrence-free patients after initial adjuvant therapy for 5 years is complex. There are still no validated biomarkers identifying patients who are at increased risk of late relapse.

After 2-5 years of tamoxifen therapy in patients with higher risk, extended therapy with 5 years of tamoxifen can be administered on the basis of the ATLAS study (LoE 1a/GR A/AGO++), or 2.5-5 years of AI treatment in accordance with the MA 17 study (LoE 1a/GR B/AGO++) $[132,133]$.

The data on prolonging upfront therapy with AIs beyond a total of 5 years are currently heterogeneous. While 2 studies have shown a benefit (MA.17R: 5 years of AI after 5 years of AI, with or without prior therapy with tamoxifen; LATER: 5 years of AI after 4 years of endocrine therapy, $11.7 \%$ were pretreated with $\mathrm{AI}$ and $36.9 \%$ with tamoxifen followed by AI) $[134,135]$. The recent publication of the IDEAL trial [136] and the DATA trial [137] failed to demonstrate the range of benefit that was shown in the MA.17 trial. Thus, in both trials (IDEAL, DATA), postmenopausal women received an $\mathrm{AI}$ as part of their initial adjuvant therapy. In summary, the indication for EAT using an AI after 2-5 years of tamoxifen is based on the risk of recurrence, the tolerability of the initial therapy, good bone health, and younger age. This needs to be discussed on an individual basis.

Three studies presented at the last San Antonio Breast Cancer Symposium were negative for the overall groups (DATA, IDEAL, and National Surgical Adjuvant Breast and Bowel Project (NSABP) B-42). However, since subgroups can possibly benefit, extension of endocrine therapy with an AI should be offered after initial AIcontaining therapy in patients who are at higher risk and have so far tolerated the AI well - e.g., those with good bone health, younger age, high risk according to immunohistochemical characteristics, and positive nodal status ( $\mathrm{LoE}$ 1b/GR B/AGO+). However, there is as yet no evidence of a significant effect on OS. In patients who are at low individual risk and/or have poor tolerance for the AI, AI therapy should not be continued beyond 5 years (LoE 1b/GR B/AGO-).

\section{Adjuvant Cytotoxic and Targeted Therapy}

If adjuvant chemotherapy is indicated, neoadjuvant therapy should always be considered $(\mathrm{AGO}++)$. In adjuvant therapy, systemic treatment encompassing 6 cycles of FEC (5-fluorouracil, epirubicin, cyclophosphamide) is no longer recommended.

Standard adjuvant chemotherapy consists of combination regimens based on anthracyclines and taxanes in patients with HER2negative tumors (LoE 1a/A/AGO++). Recommended conventional regimens include $4 \times \mathrm{EC} / \mathrm{AC}$ (epirubicin, cyclophosphamide or doxorubicin, cyclophosphamide), followed by $12 \times$ paclitaxel (weekly, q7d) (LoE 2b/B/AGO++). The benefit of this regimen has recently been confirmed in the 10-year follow-up data from E1199 [138]. The study population was enriched with patients carrying high-risk features (46\% premenopausal, $88 \%$ with involved lymph nodes). There were significant advantages for paclitaxel weekly re- garding DFS and OS (DFS $p<0.001$, OS $p=0.07$ ). Of particular interest was the subgroup with TNBC: After 4 cycles of AC, paclitaxel weekly showed a significant OS benefit in comparison with 3-weekly paclitaxel (HR 0.69, 95\% CI 0.50-0.94).

In patients with cardiac comorbidities, treatment with docetaxel and cyclophosphamide might be used as an alternative (LoE $1 \mathrm{~b} / \mathrm{B} /$ AGO+). The recently presented results of the Plan B trial confirmed an equivalent efficacy for 6 cycles of docetaxel and cyclophosphamide compared with $4 \times$ EC followed by $4 \times$ docetaxel $(\mathrm{LoE} 1 \mathrm{~b} / \mathrm{B} / \mathrm{AGO}+)$. In individual cases, treatments using paclitaxel mono weekly (LoE 1b/B/AGO+/-) or CMF (cyclophosphamide, methotrexate, fluorouracil) (LoE 1a/A/AGO+/-) may also be considered $[139,140]$.

Dose-dense chemotherapy is recommended to be used in highrisk patients independent of tumor burden ( $\mathrm{LoE} 1 \mathrm{a} / \mathrm{A} / \mathrm{AGO}++)$. The EBCTCG meta-analysis showed a significant benefit in DFS and breast cancer mortality for dose-dense chemotherapy compared with standard dose regimens [141].

In case of 4 or more affected lymph nodes, dose-dense and dose-escalated treatment with epirubicin $\left(150 \mathrm{mg} / \mathrm{m}^{2}\right)$ followed by paclitaxel $\left(225 \mathrm{mg} / \mathrm{m}^{2}\right)$ followed by cyclophosphamide $(2,500 \mathrm{mg} /$ $\mathrm{m}^{2}$ ), q14d, should be considered instead of standard 3-weekly regimens (LoE 1b/A/AGO++) [142]. At present, platinum cannot be recommended in the adjuvant setting due to a lack of data and should be considered only in individual cases (LoE 5/D/AGO+). This is in contrast to the recommendations in the neoadjuvant setting (see above).

In HER2-positive disease, an anti-HER2-directed treatment with trastuzumab is standard ( $\mathrm{LoE} 1 \mathrm{a} / \mathrm{A} / \mathrm{AGO}++$ ). In case of a positive nodal status and/or HR negativity, the application of a dual blockade consisting of trastuzumab and pertuzumab is recommended according to the data of the APHINITY trial (node positive: HR 0.77, 95\% CI, 0.62-0.96, $\mathrm{p}=0.02$; HR negative: HR 0.76, 95\% CI 0.56-1.04, $\mathrm{p}=0.0847)(\mathrm{LoE} 1 \mathrm{~b} / \mathrm{B} / \mathrm{AGO}+)$ [143].

Trastuzumab with or without pertuzumab is recommended simultaneously in combination with a taxane (LoE $1 \mathrm{~b} / \mathrm{A} / \mathrm{AGO}++)$. The optimal duration is 1 year ( $\mathrm{LoE} 1 \mathrm{~b} / \mathrm{A} / \mathrm{AGO}++)[144,145]$.

Trastuzumab subcutaneously (s.c.) has the same efficacy as the intravenous (i.v.) formulation and can be used without concerns (LoE 1b/B/AGO++) [146, 147].

Alternative anthracycline-free combination partners for trastuzumab are docetaxel and carboplatin (LoE $1 \mathrm{~b} / \mathrm{A} / \mathrm{AGO}+$ ) or, in individual cases, e.g. in patients with tumors $<2 \mathrm{~cm}$ and negative nodal status, treatment with $12 \times$ paclitaxel q7d (LoE 2b/B/AGO+) [148].

After 1 year of trastuzumab, an extension of the adjuvant antiHER2 treatment with 1 year of neratinib can be recommended after individual risk/benefit discussion with the patient. In HR-positive disease, the ExteNET trial showed a significantly increased DFS of $91.2 \%$ versus $86.8 \%$ (HR $0.60,95 \%$ CI $0.43-0.83$ ); however, diarrhea G3 occurred in $40 \%$ of the patients (LoE 2b/B/AGO+/) [202].

Biosimilar trastuzumab has become a reality for European oncologists. Several studies are still ongoing, but 2 trastuzumab biosimilars are already approved by the European Medicines Agency (EMA). These compounds can be used in daily practice if they have 
passed a stringent development and validation process required by the EMA, the US Food and Drug Administration (FDA), or other similarly strict authorities [149-152].

\section{Adjuvant Radiotherapy}

Adjuvant radiotherapy is an essential part of the primary treatment in early breast cancer and contributes substantially to disease control. Experts from the field of gynecology and radiation oncology representing their corresponding guideline committees, the AGO and the Deutsche Gesellschaft für Radioonkologie (German Society of Radiation Oncology/DEGRO), developed the joint AGO recommendations for adjuvant radiotherapy based on an intense consensus discussion. For technical details on radiotherapy, we agreed to refer to the corresponding updated DEGRO practical guidelines $[153,154]$.

The AGO and DEGRO experts agreed with regard to future developments in radiotherapy towards a risk-adapted approach: In many situations, radiotherapy will be optimized, reduced or even spared. On the other hand, use of radiotherapy may be established or expanded for indications that were not considered before.

The optimal type of breast irradiation after BCT is still a matter of debate. After the convincing data of the START A and START B trials as well as the Canadian trial, hypofractionated irradiation consisting of 15 or 16 fractions to total doses of $40-42$ Gy is widely accepted as the new standard of breast radiotherapy according to the German S3 guideline (www.awmf.org/leitlinien/detail/ll/032045OL.html), international guidelines [155-158], and common practice [159-161], and was recently confirmed by an updated Cochrane analysis. We agreed to underscore hypofractionated irradiation (15-16 fractions) as the preferred type of irradiation and to leave conventionally fractionated radiotherapy as an alternative method. In patients $<50$ years of age and in high-risk patients aged $\geq 50$ years, an additional boost of $10-16$ Gy to the tumor bed is recommended, although the improvement in local control is quite small in patients older than 40 years [162].

If radiotherapy of the regional lymph nodes is included, conventionally fractionated radiotherapy (25-28 fractions) is still recommended.

In patients with a life expectancy below 10 years, omission of radiotherapy is an option for patients with low risk of recurrence such as pT1 pN0 R0 HR-positive/HER2-negative if adjuvant endocrine treatment is performed ( $\mathrm{LoE} 1 \mathrm{a} / \mathrm{B} / \mathrm{AGO}+)$ [163]. There is no influence on OS, and side effects can be avoided. The AGO and the DEGRO agree that in elderly low-risk patients accelerated partial breast irradiation (APBI) can be delivered as the sole radiotherapy modality either during surgery (intraoperative radiation therapy (IORT) $50 \mathrm{kV}$, intraoperative electron radiation therapy (IOERT)) or after surgery using brachytherapy (patients $>50$ years, only for pT1 pN0 R0 G1-2, HR-positive, non-lobular, no extensive DCIS).

Irradiation of the chest wall and regional lymphatics (post-mastectomy radiotherapy, PMRT) is indicated if more than 3 axillary lymph nodes were tumor-infiltrated [164]. With regard to patients with 1-3 infiltrated nodes ( $\mathrm{pN} 1$ ), we recommend PMRT for any number of positive lymph nodes in all high-risk patients and in selected patients with intermediate risk. However, retrospective analyses suggest that in low-risk patients with less than 4 tumor-infiltrated lymph nodes (pN1a), in some cases, no real benefit can be expected, e.g. in those with ER-positive, HER2-negative, well-differentiated (G1) pT1 tumors. On the other hand, in patients with high-risk features, e.g. with high axillary tumor load (i.e. $>25 \%$ of removed lymph nodes are positive), undifferentiated (G3) tumors, triple-negative immunohistochemistry, lymphovascular invasion, or in younger patients with ER-negative tumors ( $<45$ years) or HER2-positive tumors ( $<40$ years), several analyses show an elevated risk of recurrence and consequently an indication for PMRT.

Based on retrospective data, omission of PMRT was discussed in patients with pN1 tumors if 3 of 4 low-risk criteria are fulfilled (ERpositive, G1, HER2-negative, pT1) [165, 166]; on the other hand, in patients with high-risk criteria such as vessel invasion, HER2 positivity, high grade (G3), high proportion of positive lymph nodes ( $>25 \%)$, and young age $(<40$ or $<45$ years if ER-negative or medial tumor location), a benefit from PMRT is expected [166, 167]. However, for some patients, individual discussion will be required.

Radiotherapy of the medial supra-/infraclavicular and internal mammary chain lymph nodes consistently improved the DFS and distant metastasis-free survival in 2 large randomized controlled trials $[168,169]$ and in a Danish population-based study [170], resulting in a small but statistically significant OS benefit in the metaanalyses of these trials [171]. The majority of patients in these trials had either node-positive breast cancer or centrally or medially located node-negative breast cancer. The AGO and DEGRO experts recommend lymph node irradiation of the internal mammary chain, starting in high-risk patients with pN1a (G2-3, HR-negative, postmenopausal: central/medial location, $<45$ years also lateral location) or more positive lymph nodes. For patients who receive trastuzumab, radiotherapy of the internal mammary chain lymph nodes should only be performed with special emphasis on heart dose.

Currently, prospective evidence regarding PMRT following NACT and individualization based on treatment response is scarce until results from NSABP B-51 are available. PMRT is therefore recommended for all patients with initially locally advanced tumors and for initially node-positive patients without pCR. Individualization can be performed in patients with $\mathrm{cT} 1 / 2 \mathrm{cN}+$ tumors showing a pCR (ypT0/ypN0) after NACT based on the presence of risk factors (e.g. G2-3, HR-negative, premenopausal, medial/central localization).

\section{Gynecological Issues in Breast Cancer Patients/ Contraception}

\section{Treatment of Menopausal Symptoms}

Classical hormonal replacement therapy to alleviate menopausal symptoms is not indicated in breast cancer patients, particularly in ER-positive disease (LoE 1b/B/AGO-), but might be considered in individual cases and after failure of other non-hormonal treatments 
( $\mathrm{LoE} 3 \mathrm{~b} / \mathrm{B} / \mathrm{AGO}+/-$ ). Tibolone is contraindicated ( $\mathrm{LoE} 1 \mathrm{~b} / \mathrm{A} /$ AGO-/-), while topical vaginal application of low-dose estriol may be used for urogenital symptoms (LoE 4/D/AGO+/-). Menopausal symptoms such as hot flushes, night sweats, or sleep disturbances may be treated with various non-hormonal remedies, e.g. serotonin reuptake inhibitors (i.e. venlafaxine ( $\mathrm{LoE} 1 \mathrm{a} / \mathrm{A} / \mathrm{AGO}+)$ ), which carry the potential to reduce hot flushes by about $60 \%$.

The majority of studies on the efficacy of herbal treatments for menopausal symptoms - mostly hot flushes - were not conducted in women with breast cancer, and many were of short duration. Increased pharmacovigilance for herbal medicines is required, e.g. initiatives to stimulate reporting of suspected adverse reactions. Neither flax seed nor black cohosh (Cimicifuga racemosa) nor St. John's wort nor ginseng root nor soy could improve menopausal symptoms.

Physical exercise and cognitive behavioral therapy have positive effects on menopausal symptoms and, to a lesser degree, on the sexuality and physical functioning of patients with breast cancer experiencing treatment-induced menopause (LoE $1 \mathrm{~b} / \mathrm{B} / \mathrm{AGO}+$ ). Mindbody medicine (MBM; relaxation training, yoga, hypnosis) is reported to result in a moderate and even significant improvement in hot flushes scores, joint pain, fatigue, sleep, mood, and relaxation $(\mathrm{LoE} \mathrm{b} / \mathrm{B} / \mathrm{AGO}+/+)$. These effects are seen even after longer periods of application and some months after stopping MBM. There is contradictory data about the effect of acupuncture on hot flushes, but in a review comparing 12 randomized controlled trials, the authors concluded that acupuncture had no significant effect on reducing hot flushes (LoE 1a/A/AGO+/-) [172]. Acupuncture might be used to treat AI-induced joint pain ( $\mathrm{LoE} 1 \mathrm{~b} / \mathrm{B} / \mathrm{AGO}+)$ [173].

\section{Fertility Preservation}

Counseling on fertility preservation is suggested in all patients who wish to retain their fertility (LoE 4/C/AGO+). Application of $\mathrm{GnRH}$ analogues given 2 weeks prior to chemotherapy has been shown to give a higher rate of recovery of ovarian function after 2 years $(\mathrm{LoE} 1 \mathrm{a} / \mathrm{B} / \mathrm{AGO}+)$ and might have a moderate effect on fertility preservation ( $\operatorname{LoE} 2 \mathrm{a} / \mathrm{B} / \mathrm{AGO}+/-$ ) [174]. No negative effect with regard to prognosis could be observed independent of the HR status of the primary tumor.

Menstrual history is reliable only in women under 45 years of age. A more precise evaluation of the ovarian reserve (particularly in perimenopausal patients) may be obtained by the measurement of follicle-stimulating hormone (FSH) and estradiol levels in the peripheral blood. Low anti-Muellerian hormone levels seem to be indicative of reduced ovarian reserve and chemotherapy-related amenorrhea in chemotherapy-treated breast cancer patients [175]. An antral follicle count, defined as the sum of the follicle diameters of all follicles of $10 \mathrm{~mm}$ in both ovaries, can be easily performed at little extra cost.

\section{Contraception}

All patients of childbearing potential must be counseled about adequate contraception prior to systemic therapy since cytotoxic treatment, including endocrine therapy, by itself does not confer reliable protection against pregnancy. The majority of contraceptive measures have not been tested in women after breast cancer and hormone-free methods are the first choice for patients with breast cancer.

\section{Sexual Health}

Sexual complaints are common in breast cancer patients and should be assessed. They include sexual desire disorder/decreased libido (23-64\% of patients), arousal or lubrication concerns (20$48 \%$ of patients), and dyspareunia (35-38\% of patients). Screening tools may help physicians to address sexual health issues (LoE 4/C/ $\mathrm{AGO}+$ ).

Non-hormonal lubricants and moisturizers are the primary treatment for vaginal dryness. Silicone-based products may last longer than water-based or glycerin-based products (LoE 1b/B/ $\mathrm{AGO}+$ ). Microablative fractionated laser or vaginal YAG/erbium laser (erbium-doped yttrium aluminum garnet laser) may be an option for some patients to alleviate genital atrophy (LoE 2b/B/ AGO+/-) [176].

\section{Complementary Therapy - Survivorship}

'Integrative oncology is a patient-centered, evidence-informed field of cancer care that utilizes mind and body practices, natural products, and/or lifestyle modifications from different traditions alongside conventional cancer treatments. Integrative oncology aims to optimize health, quality of life, and clinical outcomes across the cancer care continuum and to empower people to prevent cancer and become active participants before, during, and beyond cancer treatment' [177].

In 2018, the AGO guidelines for 'Complementary Therapy Hormonal Treatment and Alternatives in Breast Cancer Survivors - Survivorship' did not change substantially, since the data presented in 2017 altered mostly evidence levels (LoE) but not the AGO recommendations.

Some small studies suggested that massage with or without aroma therapy may help relieve short- or medium-term pain and anxiety in people with cancer. However, the quality of evidence was very low and the results were not consistent. Acupuncture seems to significantly ameliorate menopause symptoms ( $\mathrm{LoE} 1 \mathrm{~b} / \mathrm{B} /$ $\mathrm{AGO}+$ ) without significant effect on the frequency and the severity of hot flushes ( $\mathrm{LoE} \mathrm{b} / \mathrm{B} / \mathrm{AGO}+/-)$ as a recent meta-analysis shows [178]. Also acupuncture can be recommended to reduce AI-related joint symptoms (LoE 1a/B/AGO+) [179]. A randomized blinded sham- and waitlist-controlled trial with 226 patients was presented by Dawn Hershman at the San Antonio Breast Cancer Symposium 2017.

Evidence is growing that acupressure can be considered to ameliorate cancer-related fatigue ( $\mathrm{LoE} \mathrm{b} / \mathrm{B} / \mathrm{AGO}+$ ) and insomnia $[180,181]$. Patients can be taught to apply the acupressure to themselves.

A meta-analysis of 10 studies $(n=1,709)$ revealed evidence for the short-term effectiveness and safety of mindfulness-based inter- 
ventions in women with breast cancer during and after adjuvant treatment. Compared to usual care, significant post-intervention effects were found for health-related quality of life, fatigue, sleep, stress, anxiety, and depression (LoE 1a/A/AGO+) [182].

Exercise leads to several positive outcomes in oncology. A recent randomized controlled trial showed that exercise intervention significantly decreased sleep problems in breast cancer patients going through radiotherapy (LoE 1a/A/AGO++) [183].

A Cochrane review including 24 studies with a total of 2,166 participants supports the recommendation of yoga as a supportive intervention for improving health-related quality of life and reducing fatigue and sleep disturbances as well as for reducing depression, anxiety, and fatigue, when compared with psychosocial/educational interventions (LoE $1 \mathrm{~b} / \mathrm{A} / \mathrm{AGO}+$ ) [184].

A well-designed non-inferiority trial demonstrated clinically meaningful improvements in insomnia after cognitive behavioral therapy and tai chi, a movement meditation. Tai chi was found to be statistically non-inferior to CBT-I, the gold standard for behavioral treatment of insomnia (LoE 2a/B/AGO+/-) [185].

\section{Breast Cancer: Special Situations}

Breast cancer in pregnancy should be treated as close as possible to the guidelines in non-pregnant patients [186]. Nevertheless, staging and systemic therapy do have some restrictions due to potential fetal harm. Recent cohort studies indicate that whole-body MRI without a contrast agent provides valuable staging information and may be considered in individual high-risk cases ( $\mathrm{AGO}+/-$ ) [187]. Surgery should be performed as in non-pregnant women and SLNB is feasible. Regarding systemic therapy, anthracyclines and taxanes are safe to be used in pregnancy. Platinum salts may be considered $(\mathrm{AGO}+/-)$ based on data mostly from gynecological tumors [188]. Radiotherapy, endocrine therapy, and monoclonal antibodies should be avoided during pregnancy.

In inflammatory breast cancer (IBC), mastectomy is standard of care (AGO+). In a large IBC cohort from the Surveillance, Epidemiology and End Results (SEER) data base $(n=3,374)$, no statistically significant difference regarding breast cancer-specific survival or OS was found between the different types of breast surgery such as breast-conserving surgery, contralateral prophylactic mastectomy, breast reconstruction, or standard unilateral mastectomy [189].

In axillary metastases of occult breast cancer (axillary CUP), radiotherapy of the ipsilateral breast improves outcome (AGO+). In a large case series from the National Cancer Database, axillary metastases with an occult breast cancer were very rare (0.09\%). Treatment with radiotherapy and $\mathrm{AD}$ was an independent predictor of OS in multivariable analysis (HR 0.509, 95\% CI 0.321-0.808, p = 0.004) [190].

Metaplastic breast cancer is a rare subtype with an incidence of $0.2-5 \%$ of all breast cancers [191]. These tumors are characterized by an epithelial and mesenchymal differentiation with $2-3$ differ- ent components and a high proliferation rate. These different components are the basis for the classification according to the World Health Organization (WHO) criteria [192]. More than $90 \%$ of metaplastic breast cancers are negative for ER, PR, and HER2 but, in contrast, there is an overexpression of HER 1 and cytokeratin 5/6 (CK5/6) (stem cell and BRCA-like) [193] and the molecular profile is more basal-like [194]. The clinical features show large tumors at diagnosis $(>5 \mathrm{~cm})$, frequent hematogenous metastases and nodal involvement in about $20 \%$ of all cases. Imaging and gaining histology for diagnosis should be performed according to standard $(\mathrm{AGO}++)$. Due to the high frequency of hematogenous metastases, staging should encompass chest and abdominal CT (AGO++). Surgical treatment can be performed according to standard (including SLNB, AGO+), but mastectomy is more often necessary due to more advanced tumor stage and the goal of tumor-free resection margins of more than $3 \mathrm{~cm}$ [195-197]. Adjuvant treatment consists of chemotherapy (even tumors are more chemoresistant), endocrine therapy (only in HR-positive tumors) [197], and standard radiotherapy [198].

\section{Conclusion}

These guidelines offer the freshest recommendations in the diagnosis and treatment of early breast cancer. Meanwhile, we have reached a rather high level with regard to long-term prognosis, exhibiting low rates of recurrences and deaths even after 10 and 15 years. The trend of these recommendations shows increasing optimization and individualization by reducing treatment aggressiveness if possible (e.g. less axillary surgery, hypofractionation of radiotherapy in BCT) and escalating some specific therapies modalities (e.g. irradiation of lymph node areas, dose-dense chemotherapy). Thus, following these concepts, early breast cancer is a curable disease. Having accepted some toxicity, we have to put our efforts into the reduction of side effects now.

\section{Online Supplemental Material}

Suppl. table 1. AGO grades of recommendation.

Suppl. fig. 1. Indications for SLNB indicating also the limited value of lymphoscintigraphy.

Suppl. fig. 2. An algorithm for decision-making in cases with an indication for breast reconstruction.

Suppl. fig. 3. Subtype-specific strategies for systemic treatment indicating the recommendation to add pertuzumab only among cases with increased risk.

Suppl. fig. 4. Recommendation for adjuvant therapy with trastuzumab \pm pertuzumab.

Suppl. fig. 5. Recommendations for radiotherapy following NACT.

Suppl. fig. 6. Ovarian protection and fertility preservation in premenopausal patients receiving (neo-)adjuvant chemotherapy.

Suppl. fig. 7. Recommendations for treatment of metaplastic breast cancer.

To access the supplemental material please refer to www.karger.com/? $D O I=489329$. 


\section{Disclosure Statement}

C.L. has received consultant and speaker honoraria from Amgen, Celgene, Genomic Health, GSK, Nanostring, Novartis, Pfizer, PharmaMar, Pierre-Fabre, Roche, and TEVA. C.J. has received honoraria and research grants from Novartis, Pfizer, Amgen, Roche, Genomic Health, and AstraZeneca. M.T. has received consultant and speaker honoraria from AstraZeneca, Amgen, Celgene, Genomic Health, Myriad, Neodynamics, Novartis, Pfizer, pfm Medical, Pierre-
Fabre, Roche, RTI Surgical, Serag-Wiessner, Surgiceye, Sysmex Europe, Tesaro, and TEVA. C.T. has received consultant and speaker honoraria from Amgen, AstraZeneca, Celgene, Eisai, Lilly, MSD, Nanostring, Novartis, Pfizer, Puma, Roche, and TEVA. V.M. has received speaker honoraria from Amgen, AstraZeneca, Daiichi-Sankyo, Eisai, Pfizer, Novartis, Roche, Teva, Tesaro, consultancy honoraria from Hexal, Roche, Pfizer, Amgen, Daiichi-Sankyo, Nektar and Eisai. W.J. has received honoraria and research grants from Novartis, Pfizer, Amgen, Chugai, Roche, Genomic-Health, AstraZeneca, Lilly.

\section{References}

References can be found in the appendix; please refer to www. karger.com/?DOI $=489329$. 